\title{
Impacto das micro-ondas na avaliação colorimétrica e nas características morfológicas do trigo, farinha e glúten
}

Impact of microwaves on colorimetric evaluation and morphological characteristics of wheat, flour and gluten

Impacto de las microondas en la evaluación colorimétrica y las características morfológicas del

trigo, la harina y el gluten

Recebido: 10/06/2021 | Revisado: 22/06/2021 | Aceito: 22/06/2021 | Publicado: 07/07/2021

Flávio Martins Montenegro

ORCID: https://orcid.org/0000-0003-0476-4764 Instituto de Tecnologia de Alimentos, Brasil E-mail: flavio@ital.sp.gov.br

Antonio Marsaioli Júnior

ORCID: https://orcid.org/0000-0002-4305-3466 Instituto de Tecnologia de Alimento, Brasil

E-mail: tonymars@ital.sp.gov.br

Michele Nehemy Berteli

ORCID: https://orcid.org/0000-0002-9765-1947 Instituto de Tecnologia de Alimento, Brasil E-mail: berteli@gmail.com

Marcella Aparecida Stahl

ORCID: https://orcid.org/0000-0003-1550-364X Universidade Estadual de Campinas, Brasil

E-mail: m145046@dac.unicamp.br

Ana Paula Badan Ribeiro

ORCID: https://orcid.org/0000-0002-6532-1265 Universidade Estadual de Campinas, Brasil

E-mail: anabadan@fea.unicamp.br

Pedro H. Campelo

ORCID: https://orcid.org/0000-0002-5137-0162 Universidade Federal do Amazonas, Brasil

E-mail: pedrocampelo@ufam.edu.br

Maria Teresa Pedrosa Silva Clerici

ORCID: https://orcid.org/0000-0002-8445-336X Universidade Estadual de Campinas, Brasil

E-mail: mclerici@unicamp.br

\begin{abstract}
Resumo
Os processos físicos vem sendo cada vez mais utilizados em substituição aos processos que utilizam agentes químicos para tratamento de grãos. Desta forma, o presente trabalho teve como objetivo avaliar grãos de trigo que foram submetidos ao processamento por micro-ondas nas características morfológicas dos grãos de trigo tratados, farinhas de trigo e seus respectivos glútens, através das análises de cor instrumental, microscopia óptica (MO) e microscopia eletrônica de varredura (MEV). Os resultados mostraram alterações significativas, para o teste de comparação entre as médias Scott-Knott $(\mathrm{p} \leq 0,05)$, para os trigos tratados por micro-ondas provenientes dos tratamentos à $100 \mathrm{~W} / 54 \mathrm{~min}$ (TM 1), $450 \mathrm{~W} / 18 \mathrm{~min}$ (TM 2) e $750 \mathrm{~W} / 10 \mathrm{~min}$ (TM 3), obtendo valores maiores e diferentes estatisticamente, nas regiões amarela $\left(+b^{*}\right)$ e vermelha $\left(+a^{*}\right)$, quando comparados com os mesmos parâmetros para o trigo controle (TC). As diferenças totais de cor encontradas $(\Delta \mathrm{E})$ entre os trigos tratados por micro-ondas e o trigo controle, que ficaram situados na faixa de 8,94 à 14,83, cujas diferenças totais de cor em relação aos trigos controle podem ser classificadas como muito perceptíveis e possíveis de serem diferenciadas visualmente. Nas farinhas de trigo analisadas provenientes dos tratamentos por micro-ondas à $100 \mathrm{~W} / 54 \mathrm{~min}$ (FM 1), $450 \mathrm{~W} / 18 \mathrm{~min}$ (FM 2) e 750 W/10 min (FM 3) para o parâmetro de luminosidade $\left(\mathrm{L}^{*}\right)$ de cor instrumental, sendo menores que a luminosidade da farinha de trigo controle (FC). Através da avaliação da morfologia pelas microscopias ópticas e eletrônica de varredura foi possível identificar as alterações provocadas pelo processamento por micro-ondas nas estruturas do trigo em grão, na farinha de trigo e se torna mais evidente na matriz proteica dos glútens liofilizados, que apresenta danos mais expressivos nos glútens provenientes dos trigos tratados a $450 \mathrm{~W} / 18 \mathrm{~min}$ (GM 2) e $750 \mathrm{~W} / 10 \mathrm{~min}$ (GM 3). Assim, podemos concluir que a avaliação da morfologia nos materiais estudados fornece ferramentas importantes e que complementam as determinações clássicas das análises físicas, físico-químicas e reológicas.
\end{abstract}


Palavras-chave: Micro-ondas; Colorimetria; Microscopia óptica (MO); Microscopia eletrônica de varredura (MEV); Trigo; Farinha de trigo; Glúten.

\begin{abstract}
Physical processes have been increasingly used to replace processes that use chemical agents to treat grains. Thus, this study aimed to evaluate wheat grains that were subjected to microwave processing on the morphological characteristics of the treated wheat grains, wheat flours and their respective glutens, through instrumental color analysis, optical microscopy (MO) and scanning electron microscopy (SEM). The results showed significant changes for comparison among means by Scott-Knott test ( $\mathrm{p} \leq 0.05)$, for the wheat treated by microwave from treatments at $100 \mathrm{~W} / 54 \mathrm{~min}$ (TM 1), $450 \mathrm{~W} / 18 \mathrm{~min}$ (TM 2) and $750 \mathrm{~W} / 10 \mathrm{~min}$ (TM 3), obtaining higher and statistically different values, in the yellow $\left(+b^{*}\right)$ and red $\left(+a^{*}\right)$ regions, when compared with the same parameters for the control wheat (TC). The total color differences found $(\Delta \mathrm{E})$ between microwave-treated wheat and control wheat, which were in the range of 8.94 to 14.83 , whose total color differences in relation to control wheat can be classified as very perceptible and possible to be differentiated visually. In the analyzed wheat flours from the microwave treatments at $100 \mathrm{~W} / 54 \mathrm{~min}$ (FM 1), $450 \mathrm{~W} / 18$ $\min (\mathrm{FM} 2)$ and $750 \mathrm{~W} / 10 \mathrm{~min}$ (FM 3) for the luminosity parameter ( $\mathrm{L}^{*}$ ) of instrumental color, being lower than the luminosity of the control wheat flour (FC). Through the evaluation of morphology by optical microscopy and scanning electron it was possible to identify the changes caused by microwave processing in the structures of wheat grain, in wheat flour and it becomes more evident in the protein matrix of lyophilized gluten, which presents expressive damage in gluten from wheat treated at $450 \mathrm{~W} / 18 \mathrm{~min}(\mathrm{GM} 2)$ and $750 \mathrm{~W} / 10 \mathrm{~min}$ (GM 3). Thus, we can conclude that the evaluation of the morphology in the studied materials provides important tools that complement the classic determinations of physical, physical-chemical and rheological analyzes.

Keywords: Microwave; Colorimetry; Optical microscopy (MO); Scanning electron microscopy (SEM); Wheat; Wheat flour; Gluten.
\end{abstract}

\title{
Resumen
}

Los procesos físicos vienen siendo cada vez más utilizados para sustituir a aquellos que utilizan agentes químicos para el tratamiento de granos. En ese sentido, el presente trabajo tuvo como objetivo evaluar las características morfológicas de los granos de trigo, harina de trigo, y sus respectivos glutens tratados por microondas, mediante análisis de color instrumental, microscopia óptica (MO) y microscopia electrónica de barrido (MEB). Los resultados mostraron alteraciones significativas, según el test de comparación de Scott-Knott $(\mathrm{p} \leq 0,05)$, en los granos de trigo tratados a 100 W/54 min (TM 1), $450 \mathrm{~W} / 18 \mathrm{~min}(\mathrm{TM} 2)$ y $750 \mathrm{~W} / 10 \mathrm{~min}$ (TM 3), quienes obtuvieron valores mayores y estadísticamente diferentes, en las regiones amarilla $\left(+b^{*}\right)$ y roja $\left(+a^{*}\right)$ en comparación con el trigo control (TC). Las diferencias totales del color encontradas $(\Delta \mathrm{E})$ entre los trigos tratados por microondas y el trigo control, que quedaron situados en un rango de 8.94 y 14.83, pueden ser clasificadas como muy perceptibles y posibles de diferenciar visualmente. En las harinas de trigo analizadas de los tratamientos por microondas a $100 \mathrm{~W} / 54 \mathrm{~min}$ (FM 1), $450 \mathrm{~W} / 18$ min (FM 2) e 750 W/10 min (FM 3) para el parámetro de luminosidad (L), siendo inferior a la luminosidad de la harina de trigo control. Mediante la evaluación de la morfología por microscopía óptica y electrónica de barrido fue posible identificar las alteraciones producidas por el procesamiento por microondas en las estructuras del trigo en grano, harina de trigo y se torna más evidente en la matriz proteica de los glutens liofilizados, los cuales presentaron daños más expresivos en glutens tratados a $450 \mathrm{~W} / 18 \mathrm{~min}(\mathrm{GM} 2)$ y $750 \mathrm{~W} / 10 \mathrm{~min}$ (GM 3). De esta forma, podemos concluir que la evaluación morfológica de los materiales estudiados provee herramientas importantes y que complementan las determinaciones clásicas de lo análisis físicos, fisicoquímicos y reológicos.

Palabras clave: Microondas; Colorimetría; Microscopía óptica (MO); Microscopía electrónica de barrido (MEB); Trigo; Harina de trigo; Gluten.

\section{Introdução}

As proteínas insolúveis presentes no grão de trigo, gliadina e glutenina, possuem a especial característica de formação, quando associadas com a água e trabalho mecânico, de uma rede viscoelástica a que chamamos de glúten, sendo esta a principal característica que diferencia o trigo dos demais cereais (Cauvain, 2015).

É conhecido que proteínas podem se desnaturar, perder suas características e funcionalidades tecnológicas quando submetidos a tratamentos físicos ou químicos. As proteínas do trigo formadoras do glúten são sensíveis quando submetidas a tratamentos térmicos, visto que em temperaturas acima de $55^{\circ} \mathrm{C}$ as gluteninas e gliadinas acabam sofrendo reações de quebra de pontes dissulfeto (SS) e gerando radicais sulfidrilas (SH) o que desestabiliza sua coesividade (Lamacchia, Landriscina \& D'Agnello, 2016).

Desde sua colheita, o trigo é submetido a processos que podem promover esta perda de qualidade, através das 
modificações de suas características, onde com a finalidade de se obter condições adequadas para armazenamento e proporcionar com isso extensão de vida de prateleira e minimização de perdas nutricionais, trigos com umidades superiores aos $13 \%$ passam por processo de secagem. Esta secagem pode acontecer de forma natural, onde o principal ponto negativo é o tempo necessário para atingir a umidade de segurança, ou de forma artificial, sendo o método com o emprego de altas temperaturas, um dos mais utilizados para alcançar a redução de umidade do material (Aulakh \& Regmi, 2013; Baloch, 2001; Hagstrum et al., 2012; Kumar \& Kalita, 2017).

A secagem por micro-ondas é uma das alternativas para processos convencionais pois, dentre os principais benefícios envolvidos podemos destacar o baixo impacto ambiental, por utilizar uma energia renovável, menor consumo energético, visto que demandam tempos menores de execução gerando eficiência térmica e como consequência podem melhorar a qualidade do produto, minimizando perdas durante o processamento (Bansal, Dhaliwal \& Mann, 2015; Patil, Kar \& Mohapatra, 2016; Vadivambal, Jayas \& White, 2007; Hemis, Singh \& Jayas, 2011).

As micro-ondas são caracterizadas como radiação eletromagnética que acontecem na faixa de frequência situada entre $300 \mathrm{MHz}$ e $300 \mathrm{GHz}$ (Berteli, 2005; Vadivambal et al., 2007). O aquecimento dos materiais ocorre pela absorção das microondas pelos materiais que possuem a habilidade de converter a radiação em calor, onde esta habilidade é dada o nome de potencial dielétrico. Cada material possui propriedades dielétricas específicas que vão determinar maior ou menor capacidade de conversão da radiação em calor e que são diretamente relacionadas a quantidades de umidade, sais e outros componentes (Bansal et al., 2015).

Além destes aspectos, conhecer como estas proteínas podem ser influenciadas por esta tecnologia é de fundamental interesse comercial para produtores agrícolas, empresas que o utilizam para produtos de panificação e fabricantes de glúten e amido (Lamacchia, Landriscina \& D'Agnello, 2016).

Desta forma, o presente trabalho objetivou avaliar os aspectos morfológicos do trigo em grão, comportamento das proteínas do glúten e grânulos de amido que foram submetidos a processamento por micro-ondas através da análise de cor, microscopia óptica (MO) e microscopia eletrônica de varredura (MEV), como avaliações complementares às determinações clássicas realizadas.

\section{Metodologia}

\subsection{Matéria-prima e tratamento por micro-ondas}

O trigo (Triticum aestivum, L.) utilizado no presente estudo foi produzido na região sul do Brasil, na cidade de Arapongas-PR, com coordenadas geográficas $23^{\circ} 25^{\prime} 10^{\prime \prime}$ latitude sul e $51^{\circ} 25^{\prime} 28^{\prime \prime}$ longitude oeste. Em sua caracterização inicial, o trigo apresentou umidade de $11,28 \pm 0,05 \mathrm{~g} / 100 \mathrm{~g}$ de umidade, $13,37 \pm 0,13 \mathrm{~g} / 100 \mathrm{~g}$ de proteína, $80 \mathrm{~kg} / \mathrm{hL}$ de peso hectolítrico $(\mathrm{PH})$ e $78 \%$ de seus grãos foram classificados como duros, pelo grau de dureza através da análise de Single-Kernel Characterization System - SKCS (AACCI, 2010).

O condicionamento das amostras seguiu a metodologia AACCI nº 26-10.02 e 26-95.01 (AACCI, 2010), onde para o trigo controle (TC), elevou-se a umidade do grão de trigo para $16 \%$, recomendada para trigos duros, previamente a etapa de moagem. Para os trigos que sofreram tratamento por micro-ondas (TM), a umidade foi elevada para $23 \%$, para atingirem os $16 \%$ após o processamento por micro-ondas.

O processamento do trigo em grão foi realizado em equipamento piloto multifuncional destinado ao estudo da aplicação de micro-ondas em alimentos com as seguintes características: $2,45 \mathrm{GHz}$ de frequência, variação de potência de $100 \mathrm{~W}$ a 3000 W transmitidas ao interior da cavidade. As potências transmitidas e refletidas foram medidas através de dois wattímetros, acoplados ao sistema de transmissão de micro-ondas, com capacidade para medição de até $3 \mathrm{~kW}$ (Clerici et al., 2019). Lotes de $600 \mathrm{~g}$ de trigo, foram depositados em um porta amostra, com $27 \mathrm{~cm}$ de diâmetro, produzido com material inerte às micro-ondas, 
posicionado no interior da cavidade receptora das micro-ondas e foram aplicadas as potências de $100 \mathrm{~W}, 450 \mathrm{~W}$ e $750 \mathrm{~W}$ às amostras, pelos tempos de $54 \mathrm{~min}, 18 \mathrm{~min}$ e $10 \mathrm{~min}$, respectivamente, gerando as amostras TM 1 (100 W/54 min), TM 2 (450 $\mathrm{W} / 18 \mathrm{~min}$ ) e TM 3 (750 W/10 min), que foram comparadas com a amostra que não foi tratado por micro-ondas (TC).

\subsection{Moagem do trigo}

A moagem dos trigos foi realizada em moinho Quadrumat Jr. (Brabender, Duisburg, Alemanha), segundo método AACCI $n^{\circ}$ 26-50.01 (AACCI, 2010), produzindo as farinhas FC, FM 1, FM 2 e FM 3 (farinha controle e tratadas com 100 W/54 $\min , 450 \mathrm{~W} / 18$ min e $750 \mathrm{~W} / 10 \mathrm{~min}$, respectivamente).

\subsection{Avaliação colorimétrica e morfológica do trigo em grão, farinha de trigo e seus respectivos glútens}

\subsubsection{Cor instrumental}

A análise de cor instrumental nos trigos e farinhas deste trabalho utilizou o sistema CIELab, com valores de $\mathrm{L}^{*}, \mathrm{a}^{*}$ e $\mathrm{b}^{*}$ determinados a partir de colorímetro Minolta Chroma Meter CR-410 (Konica Minolta Inc., Tóquio, Japão). As condições para obtenção das análises foram a calibração do equipamento com placa branca padrão, iluminante padrão D65, simulando a luz do dia, e observador padrão de $10^{\circ}$. Os valores foram obtidos a partir de dez repetições para cada amostra.

\subsubsection{Morfologia}

A análise de morfologia foi realizada através das microscopias ópticas (MO) e microscopia eletrônica de varredura (MEV) nos grãos de trigo (TC, TM 1, TM 2 e TM 3), farinhas de trigo e em seus respectivos glútens, preparados a partir das farinhas FC, FM 1, FM 2 e FM 3, em equipamento Glutomatic (Perten Instrumens, Hägersten, Suécia), de acordo com a metodologia para formação de glúten úmido AACCI 38-12.02 e que posteriormente foram liofilizadas com vácuo de $50 \mathrm{mmHg}$ e temperatura de $-45^{\circ} \mathrm{C}$, em liofilizador modelo LS 3000 (Terroni, São Carlos-SP, Brasil) (AACCI, 2010).

\subsubsection{Microscopia Óptica}

As farinhas de trigo FC, FM 1, FM 2 e FM 3 e seus respectivos glútens liofilizados GC, GM 1, GM 2 e GM 3, foram observados em microscópio de luz polarizada, modelo BX41 (Olympus Corp., Tóquio, Japão). As imagens das farinhas foram obtidas com luz padrão (luz branca) e polarizada através de câmera digital Q-Color3 (Olympus Corp., Tóquio, Japão) adaptada ao microscópio e utilizou-se software Q-capture (Olympus Corp., Tóquio, Japão), para captação e tratamento das imagens.

Para a obtenção das imagens dos glútens liofilizados, usou-se luz padrão gerada a partir de fonte de luz externa Olympus, modelo LGPS (Olympus Corp., Tóquio, Japão).

\subsubsection{Microscopia Eletrônica de Varredura (MEV)}

As superfícies das amostras dos trigos, suas farinhas e seus respectivos glútens foram visualizadas por microscopia eletrônica de varredura (MEV), em microscópio TM3000 (Hitachi High-Technologies Corp., Tóquio, Japão). As amostras de trigo em grão foram cortadas longitudinalmente e transversalmente e os glútens foram cortados em pequenos filetes. Ambos foram colocados em fita de carbono dupla face fixada em stub de alumínio, para sua visualização direta no microscópio. As farinhas de trigo foram dispersas na superfície da fita de carbono para obtenção das imagens que obtidas com magnitude de 50x a 500x e voltagem de aceleração de $15 \mathrm{kVA}$ (Analy mode).

\subsection{Análise estatística}

Os resultados de cor, obtidos a partir de pelo menos dez repetições analíticas, tiveram a análise de variância (ANOVA) 
das suas médias realizada no software SISVAR 5.6 Build 86, calculada para $\mathrm{p} \leq 0,05$ (Tabelas B.1 até B.8, do Apêndice B). Quando significativo, o teste de agrupamento de médias (Scott-Knott) foi utilizado para se determinar diferenças estatísticas entre si. A escolha pelo teste de Scott-Knott se deu pelo fato de que são excluídas médias pareadas, permitindo determinar a diferenciação entre os tratamentos através da estatística de verossimilhança (Ferreira, 2011).

\section{Resultados e Discussão}

\subsection{Cor instrumental}

$\mathrm{Na}$ avaliação da cor para o trigo em grão, pode-se observar, pelas imagens na Figura 1 e pelos resultados analíticos expressos na Tabela 1, que todos os trigos tratados e controle apresentaram diferença significativa através da análise de variância (ANOVA), $\mathrm{p} \leq 0,05$, para o parâmetro luminosidade ( $\left.\mathrm{L}^{*}\right)$, onde apresentaram valores que variaram de 61 a 71 , entretanto as amostras dos trigos tratados por micro-ondas obtiveram valores maiores e diferentes estatisticamente, para o teste de agrupamento de médias Scott-Knott $(\mathrm{p} \leq 0,05)$, nas regiões amarela $\left(+\mathrm{b}^{*}\right)$ e vermelha $\left(+\mathrm{a}^{*}\right)$, quando comparados com os mesmos parâmetros para o trigo controle. As diferenças encontradas são normais de acontecerem para grãos e materiais particulados desuniformes, pois no caso de grãos, com diferentes comprimentos, larguras e diâmetro, para a mesma replicata analisada ocorrem variações devido a heterogeneidade do material, evidenciado pelos valores da diferença total de cor $(\Delta \mathrm{E})$ entre os trigos tratados por micro-ondas e o trigo controle, que ficaram situados na faixa de 8,94 à 14,83, cujas diferenças totais de cor em relação aos trigos controle podem ser classificadas como muito perceptíveis e possíveis de serem diferenciadas a olho nu (Hidalgo et al., 2017).

Figura 1. Imagens dos trigos TC (trigo controle), TM 1 (tratamento $100 \mathrm{~W} / 54 \mathrm{~min}$ ); TM 2 (tratamento $450 \mathrm{~W} / 18 \mathrm{~min}$ ); TM 3 (tratamento $750 \mathrm{~W} / 10 \mathrm{~min}$ ).
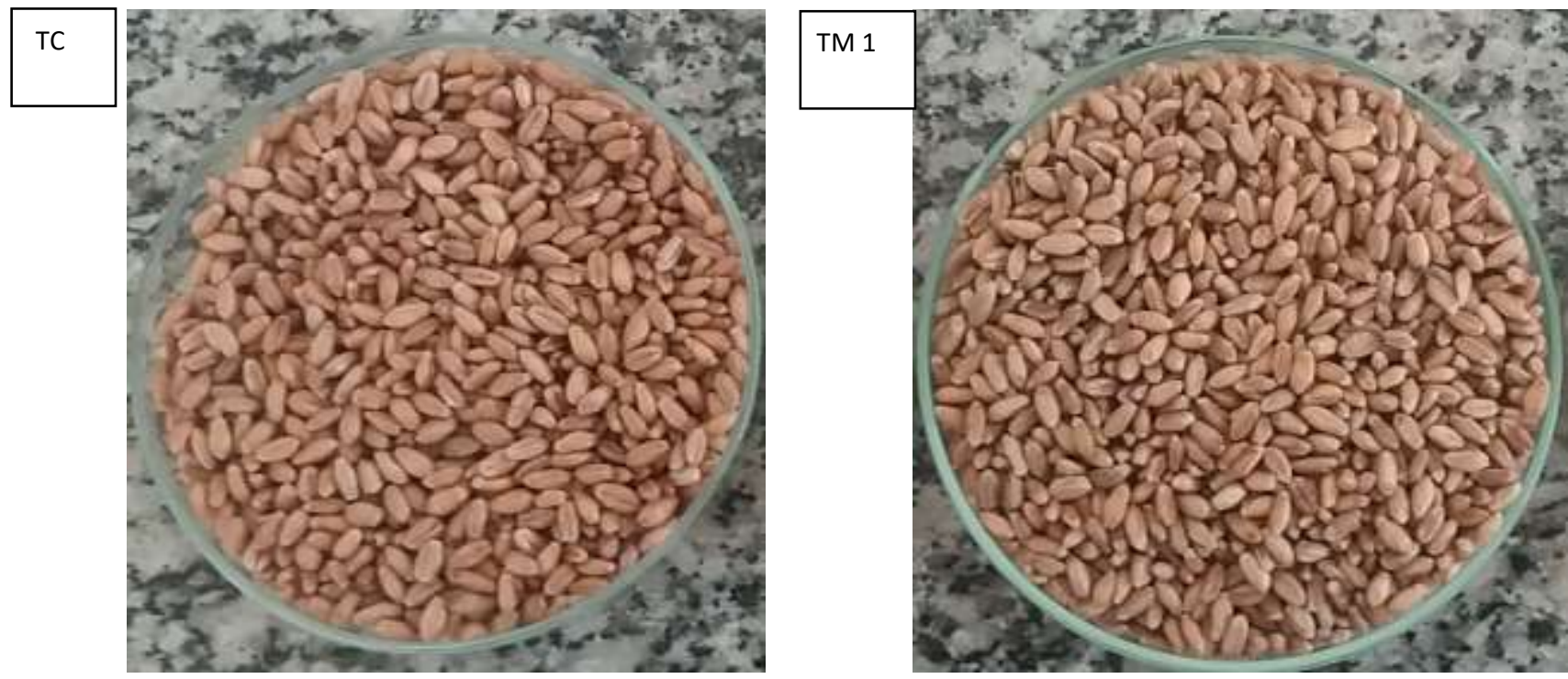

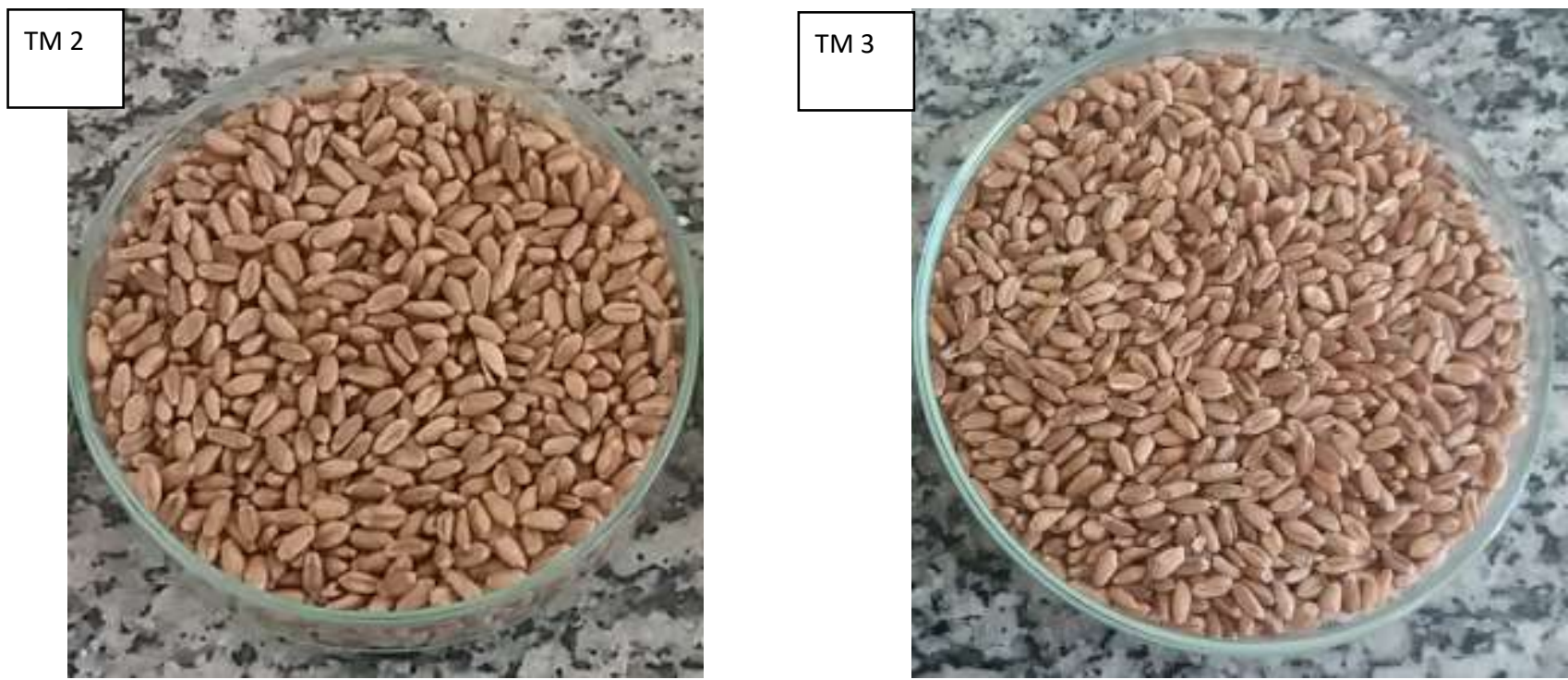

Fonte: Autores.

As farinhas de trigo analisadas apresentaram diferenças estatísticas significativas entre as amostras originadas de trigos tratados por micro-ondas, quando comparadas com a FC, que apresentou maior luminosidade (L*), 91,40 e maiores valores dos parâmetros $\mathrm{a}^{*}$ e $\mathrm{b}^{*}$, sendo $-0,31$ e 11,68, respectivamente, conforme resultados apresentados na Tabela 1. Apesar da diferença significativa para os parâmetros de cor, quando calculada a diferença total de cor entre as amostras tratadas por micro-ondas, em relação a amostra controle, sem tratamento, verifica-se que os valores foram muito baixos, situados na faixa de 1,19 e 1,44, indicando pouca variação de coloração entre as farinhas de trigo obtidas pelo estudo. Moritz (2011), Adekunte, Twari e Cullen (2010) e Hidalgo, Fongaro e Brandolini (2017) indicam que para que ocorra percepção de diferenças pelo olhar humano, os valores de $\Delta \mathrm{E}$ devem apresentar-se maiores que 2,00, conforme podemos observar a partir da Figura 2.

Figura 2. Imagens das farinhas de trigo FC (obtida de TC), FM 1 (obtida de FM 1); FM 2 (obtida de TM 2); FM 3 (obtida de TM 3).
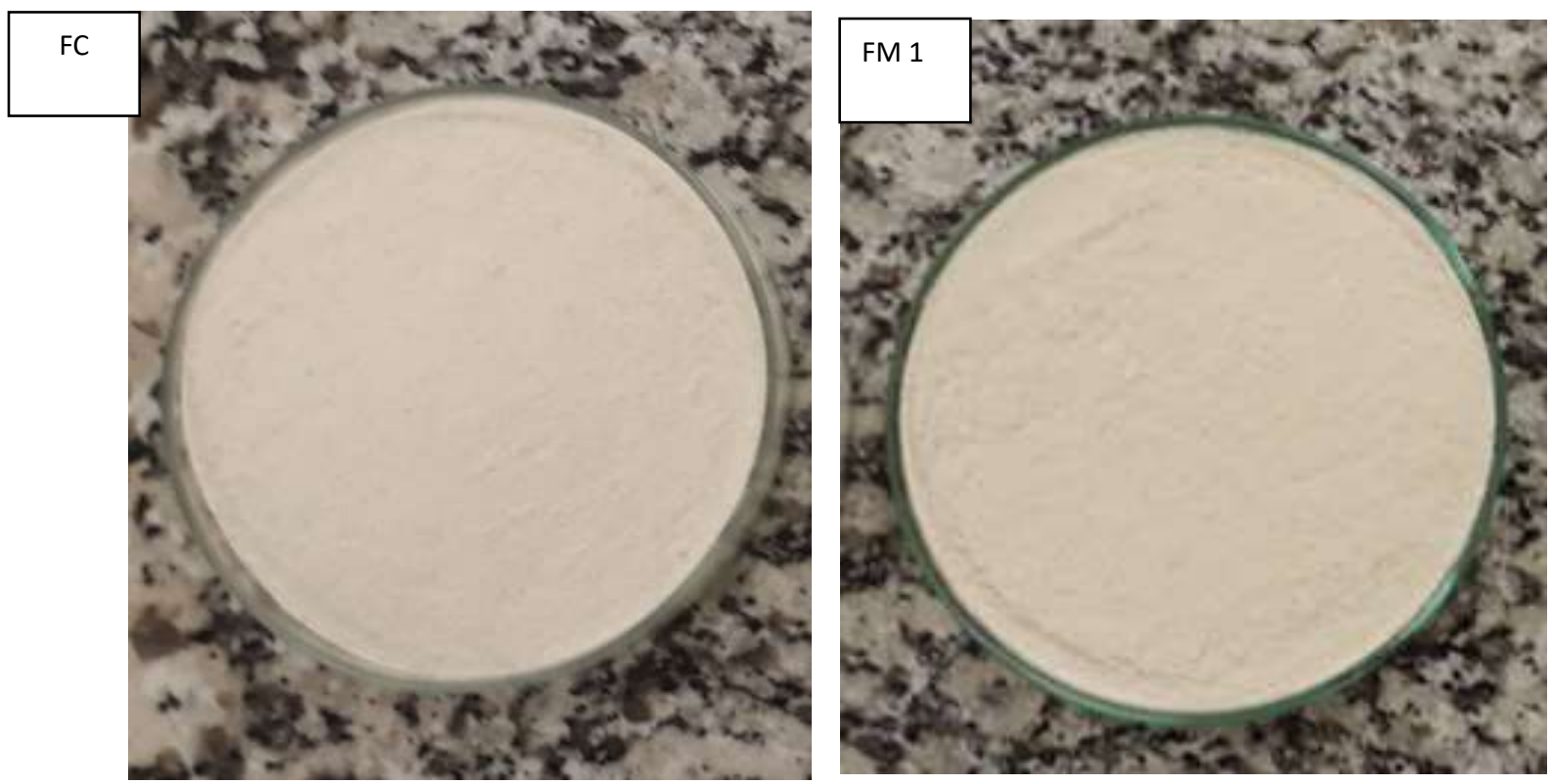

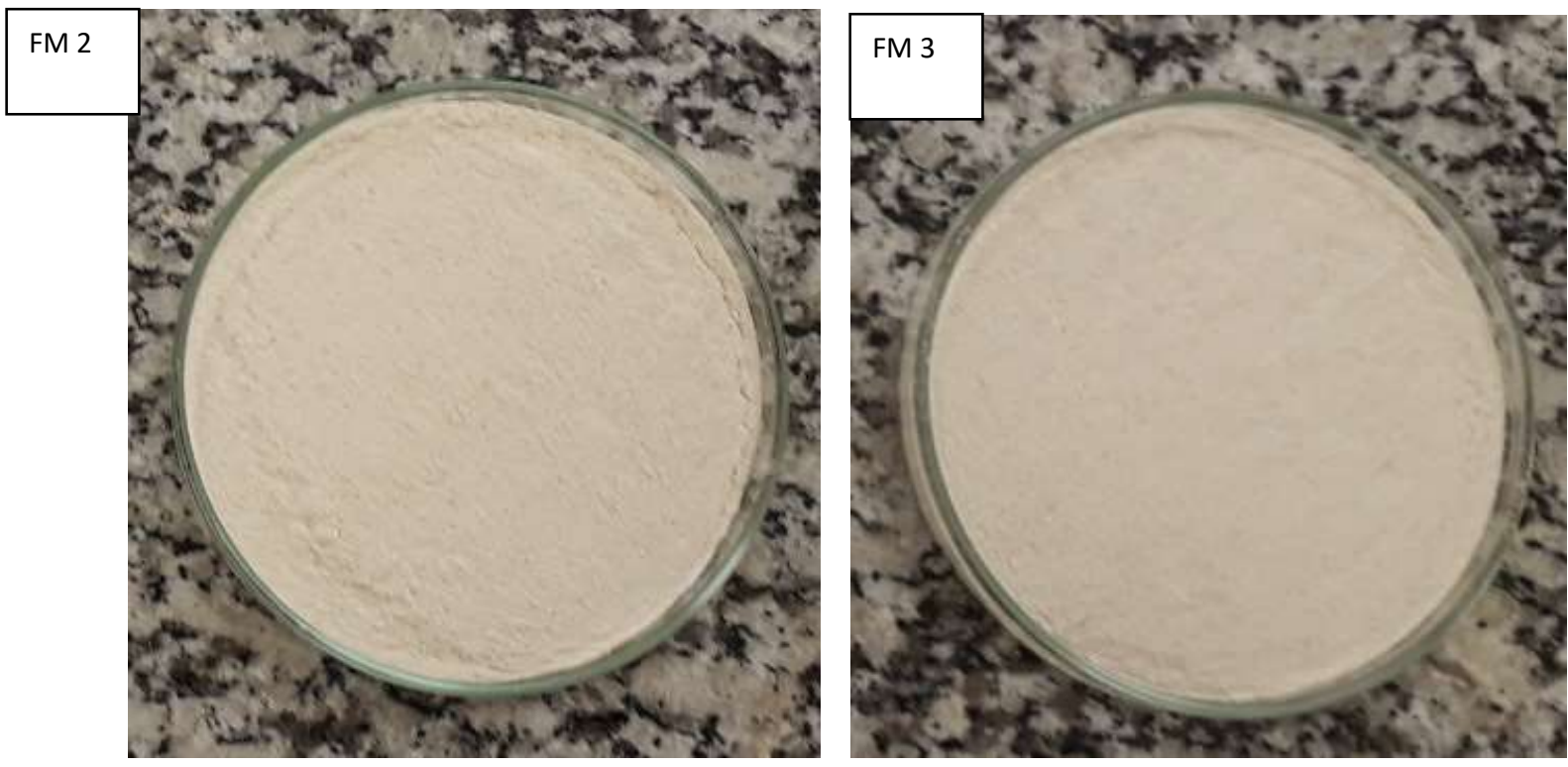

Fonte: Autores.

Tabela 1 - Cor instrumental dos grãos e farinha de trigo antes e após processamento por micro-ondas

\begin{tabular}{|c|c|c|c|c|c|}
\hline & Amostras $^{1}$ & $\mathbf{L}^{*}$ & $a^{*}$ & $\mathbf{b}^{*}$ & $\Delta \mathbf{E}$ \\
\hline \multicolumn{6}{|c|}{ Cor instrumental do trigo em grão } \\
\hline \multirow{4}{*}{$\begin{array}{c}\text { Grão de } \\
\text { trigo }\end{array}$} & $\mathrm{TC}$ & $61,29 \pm 6,08^{\mathrm{ns}}$ & $11,28 \pm 0,87^{b}$ & $27,44 \pm 2,10^{c}$ & na \\
\hline & TM 1 & $64,28 \pm 10,89^{\text {ns }}$ & $14,66 \pm 2,39^{a}$ & $32,74 \pm 2,56^{b}$ & $12,29 \pm 5,97^{\mathrm{ns}}$ \\
\hline & TM 2 & $71,34 \pm 8,26^{\mathrm{ns}}$ & $15,61 \pm 1,88^{\mathrm{a}}$ & $35,13 \pm 2,23^{\mathrm{a}}$ & $14,83 \pm 4,44^{\mathrm{ns}}$ \\
\hline & TM 3 & $63,96 \pm 8,47^{\mathrm{ns}}$ & $12,88 \pm 1,26^{\mathrm{b}}$ & $31,20 \pm 3,40^{b}$ & $8,94 \pm 4,73^{\mathrm{ns}}$ \\
\hline \multicolumn{6}{|c|}{ Cor instrumental nas farinhas de trigo } \\
\hline \multirow{4}{*}{$\begin{array}{l}\text { Farinha } \\
\text { de trigo }\end{array}$} & $\mathrm{FC}$ & $91,40 \pm 0,09^{\mathrm{a}}$ & $0,31 \pm 0,02^{\mathrm{d}}$ & $11,68 \pm 0,06^{\mathrm{a}}$ & na \\
\hline & FM 1 & $90,33 \pm 0,20^{\mathrm{b}}$ & $-0,21 \pm 0,06^{c}$ & $11,28 \pm 0,19^{b}$ & $1,19 \pm 0,23^{\mathrm{ns}}$ \\
\hline & FM 2 & $90,11 \pm 0,13^{c}$ & $-0,09 \pm 0,06^{b}$ & $11,11 \pm 0,11^{\mathrm{c}}$ & $1,42 \pm 0,12^{\mathrm{ns}}$ \\
\hline & FM 3 & $90,39 \pm 0,39^{b}$ & $0,01 \pm 0,12^{\mathrm{a}}$ & $10,74 \pm 0,09^{d}$ & $1,44 \pm 0,21^{\mathrm{ns}}$ \\
\hline
\end{tabular}

TC - trigo controle; TM 1: trigo tratado por $100 \mathrm{~W} / 54 \mathrm{~min}$; TM 2: trigo tratado por $450 \mathrm{~W} / 18$ min; TM 3: trigo tratado por 750 W/10 min; FC - farinha controle; FM 1: farinha obtida de TM 1.; FM 2: farinha obtida de TM 2.; FM 3: farinha obtida de TM 3. Resultados apresentados como média \pm desvio padrão; Médias seguidas pela mesma letra na mesma linha não diferem significativamente $(\mathrm{p} \leq 0,05)$ pelo teste de Scott-Knott; ns: sem diferença significativa pela análise de variância (ANOVA); na: não aplicável. Fonte: Autores.

\subsection{Características morfológicas do grão, farinhas de trigo e seus respectivos glútens}

\subsubsection{Efeito da radiação por micro-ondas na morfologia do endosperma dos grãos de trigo}

A radiação por micro-ondas provoca alterações significativas no comportamento reológico e perfil de viscosidade de farinhas submetidas à este tipo de tratamento térmico, consequentemente, o glúten formado, a partir destas farinhas, também sofrem alterações. As microscopias ópticas e eletrônica de varredura, foram utilizadas neste estudo para visualizar e entender estas alterações presentes nos materiais tratados por esta tecnologia.

Através das imagens obtidas pelo microscópio eletrônico de varredura, Figura 3, os trigos, seccionados transversalmente, TM 1 e TM 2, apresentam estruturas similares e preservadas, quando comparadas com a imagem do TC, 
diferente do TM 3 que apresenta suas duas extremidades, na parte inferior da imagem, aparentemente colapsadas e homogêneas, o que não aconteceu no restante do grão. Esta alteração pode ter sido provocada pela maior incidência de radiação por microondas nesta parte do grão de trigo.

Ainda na Figura 3 é possível observar as imagens do endosperma amiláceo, aleurona e subaleurona, onde identifica-se os diferentes tipos de amido presentes no endosperma, sendo os grânulos maiores (10 a $25 \mu \mathrm{m}$ ), categorizados com tipo A e os grânulos menores (2 a $10 \mu \mathrm{m}$ ), conhecidos como tipo B. Estes grânulos de amidos estão distribuídos no endosperma, ancorados nas proteínas constituintes do trigo, conforme foi possível observar nas imagens contidas na Figura 3. Este comportamento foi semelhante ao observado por Landriscina, D'Agnello e Bevilacqua (2017).

Novamente foi possível observar que as alterações foram mais evidentes no trigo TM 3, visto que ocorre um estreitamento nas células imediatamente após a camada de aleurona e subaleurona, onde os tratamentos TM 1 e TM 2 apresentamse mais íntegros e com tamanhos uniformes, além de conterem os amidos dispersos na estrutura, sem a ocorrência de espaços vazios, conforme observado na imagem do endosperma do trigo TM 3. Alguns estudos prévios indicam a possibilidade de que em altas temperaturas o endosperma amiláceo forma um agregado com a matriz proteica resultando em grandes estruturas celulares (Landriscina et al., 2017).

\subsubsection{Efeito do tratamento por micro-ondas na morfologia das farinhas de trigo e glútens}

Na Figura 4 foi possível verificar comportamento semelhante nas micrografias das farinhas de trigo FM 1, FM 2 e FM 3, quando comparadas com a farinha de trigo FC (trigo controle). As imagens obtidas pela microscopia eletrônica de varredura (MEV) dos glútens liofilizados mostram que o glúten da FC apresenta uma uniformidade da rede de glúten, com tamanhos regulares de alvéolos presentes na matriz proteica formada, indicando um glúten coeso, com boa formação da rede de glúten, típico de farinhas obtidas a partir de trigos mais fortes. O que acontece, a medida em que os trigos são submetidos aos tratamento por micro-ondas é que os glútens formados pelas farinhas de trigo FM 1, FM 2 e FM 3 apresentam danos físicos evidentes e progressivos, com rupturas da rede de glúten e formação de grandes buracos, com rupturas da matriz proteica, colapsando a estrutura formada em várias regiões presentes nas micrografias da Figura 4. 
Research, Society and Development, v. 10, n. 8, e12710817034, 2021

(CC BY 4.0) | ISSN 2525-3409 | DOI: http://dx.doi.org/10.33448/rsd-v10i8.17034

Figura 3. Micrografias dos grãos de trigo TC (trigo controle) e tratados por micro-ondas TM 1 (100 W/54 min); TM 2 (450 W/18 min) e TM 3 (750 W/10 min), observados por MEV magnitude de 50x a 500x e voltagem de aceleração de 15 kVA (Analy mode).a)secção transversal; b) secção horizontal; c) aleurona, sub-aleurona e endosperma amiláceo.

(a)
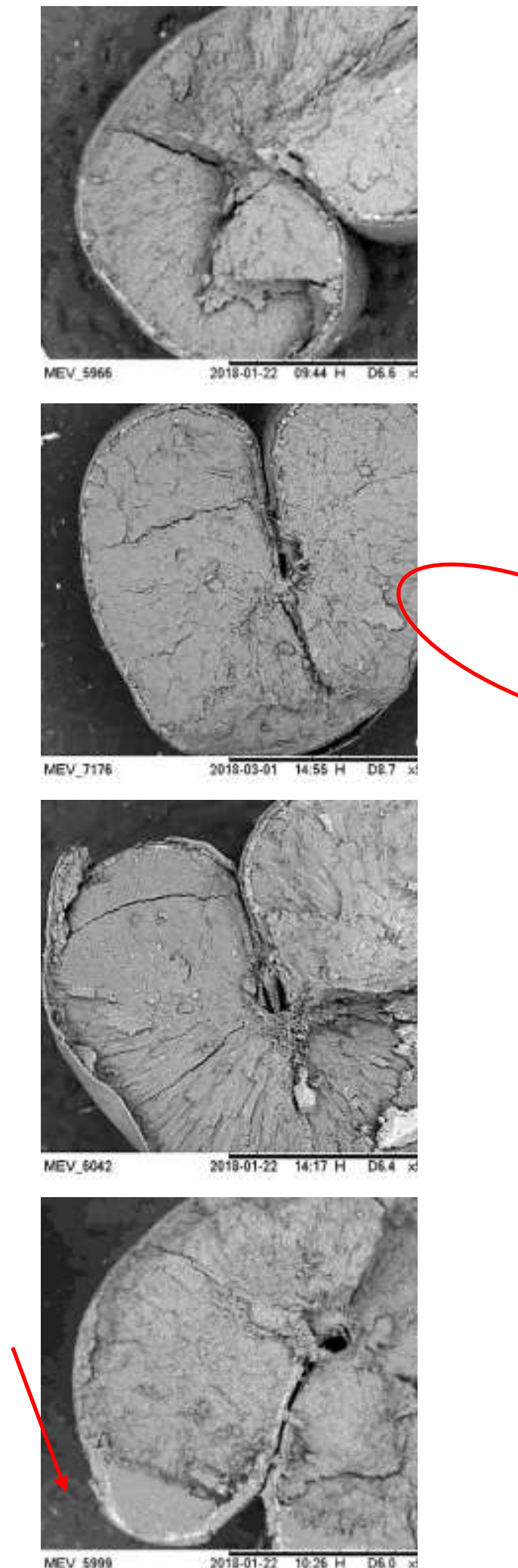

(b)
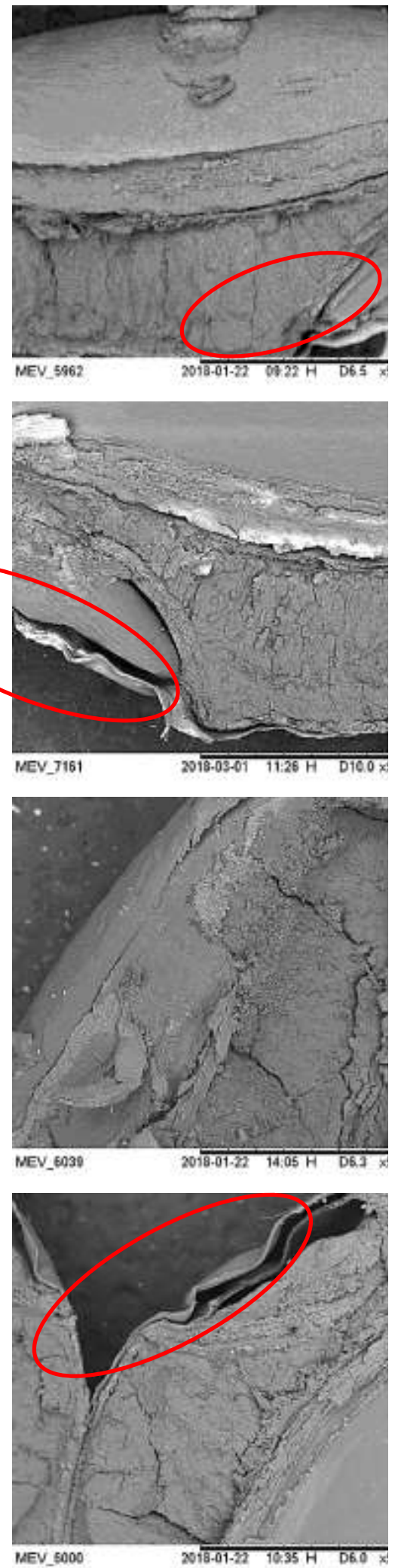

(c)
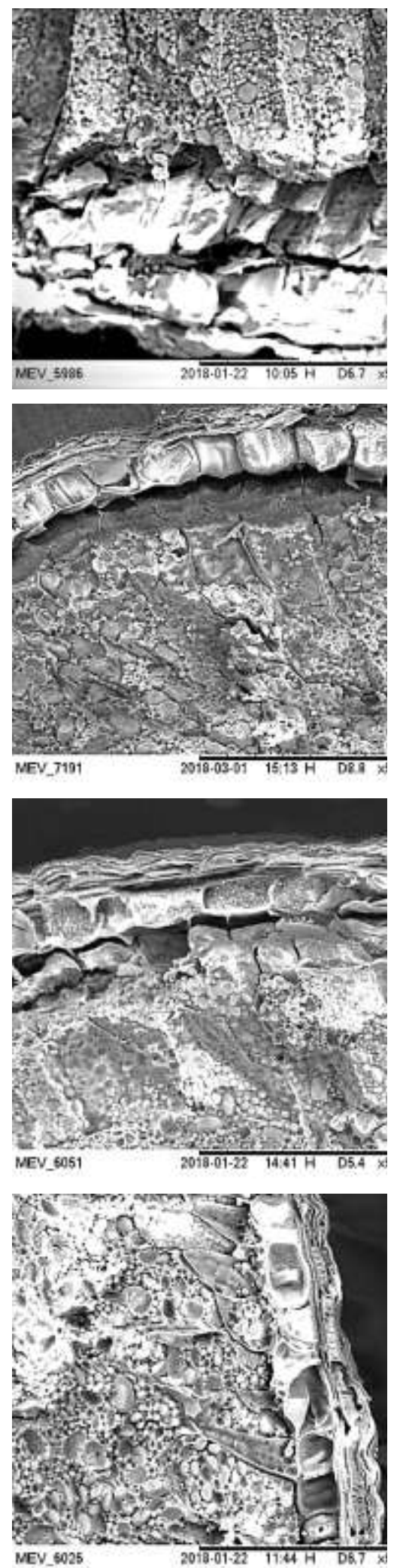
Conforme pode ser observado na figura 5, a microscopia óptica aplicada às farinhas de trigo controle e farinhas de trigo obtidas dos trigos tratados pelas micro-ondas, sob efeito de luz polarizada indicam a presença da cruz de malta, indicativo da presença de amido intacto presente na amostra e que este possui orientação radial normal em sua molécula. A medida em que foram analisadas as microscopias ópticas das farinhas obtidas dos trigos tratados por micro-ondas, foi observado o aumento de regiões com grânulos de amidos sem a presença da birrefringência, sugerindo que em decorrência do aumento da potência aplicada no tratamento térmico por micro-ondas submetidos aos trigos, danos físicos às moléculas podem ter ocorrido, assim como algum início de estágio de gelatinização acontecendo nos materiais.

Os glútens avaliados pela microscopia óptica, sob luz padrão de fonte externa, mostram a progressão dos danos causados às matrizes proteicas das redes de glútens a medida que aumentou-se a potência aplicada nos tratamentos dos trigos. O glúten controle (GC), originado de FC apresenta uma estrutura vítrea e com alvéolos uniformes da rede formada. Para os glútens GM 1, GM 2 e GM 3 (originados de FM 1, FM 2 e FM 3, respectivamente) nota-se a perda de vitreosidade e perda da estrutura uniforme, sendo que para a GM 3, o glúten apresenta-se particulado, perdendo toda a estrutura uniforme e coesa, característica apresentada para os glútens GC e GM 1. 
Figura 4. a) Micrografias dos grãos de trigo TC (trigo controle) e tratados por micro-ondas TM 1 (100 W/54 min); TM 2 (450 W/18 min) e TM 3 (750 W/10 min), b) respectivas farinhas, c) respectivos glútens, observados por MEV magnitude de 50x a 500x e voltagem de aceleração de 15 kVA (Analy mode).

(a)

TC

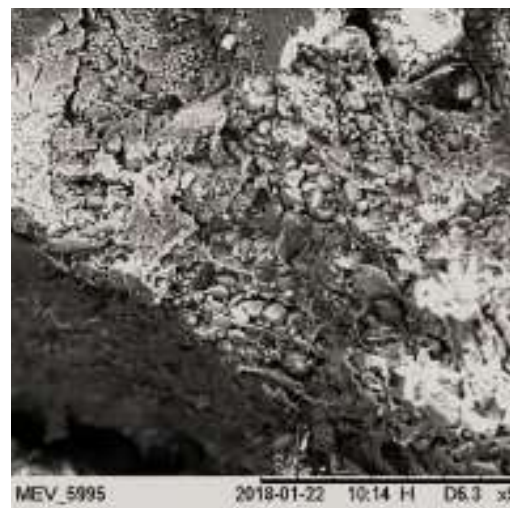

TM 1

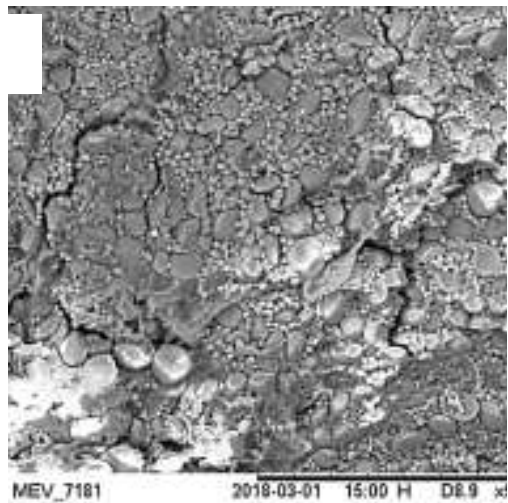

TM 2
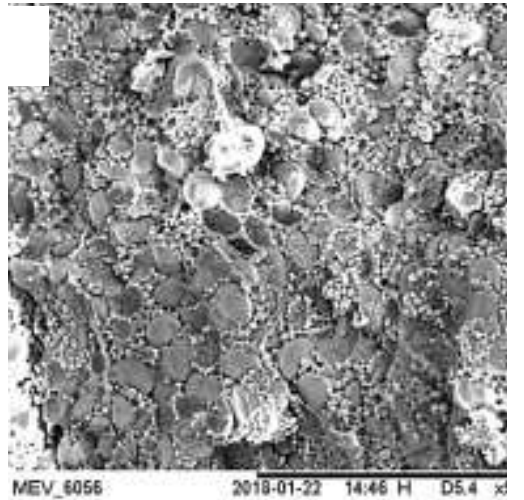

TM 3
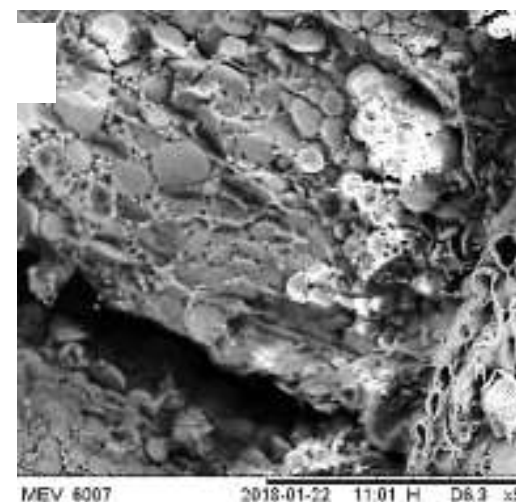

(b)

FC

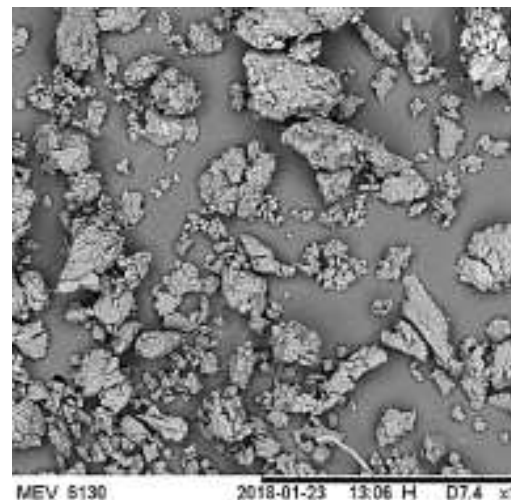

FM 1

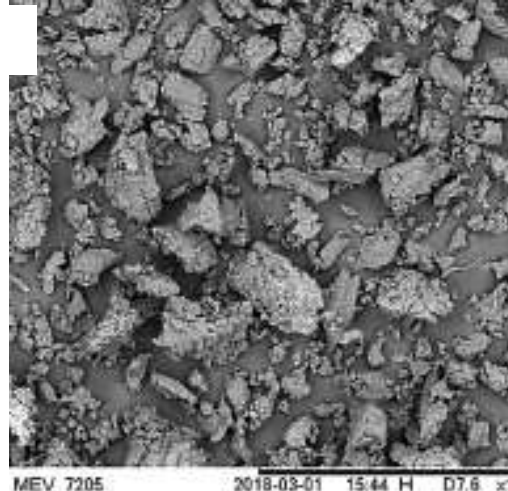

FM 2
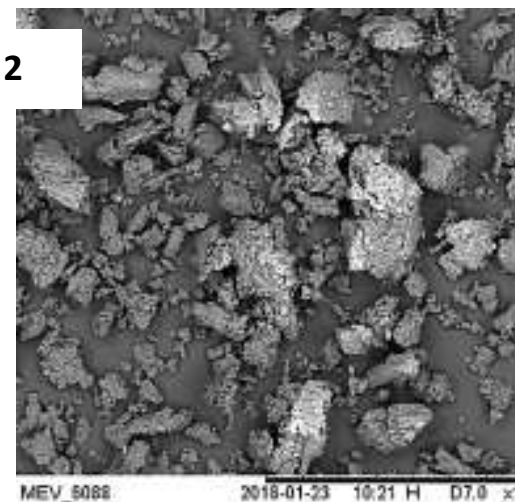

FM 3

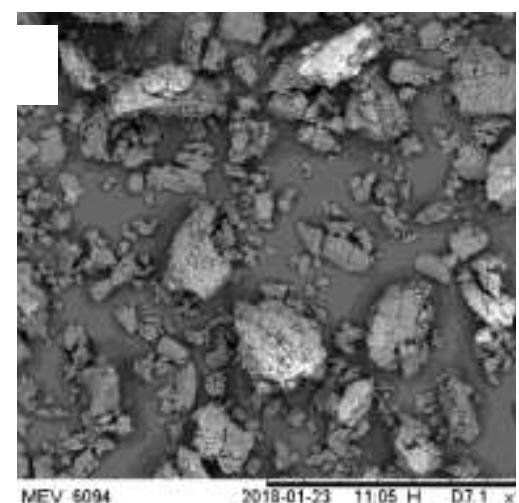

(c)

GC

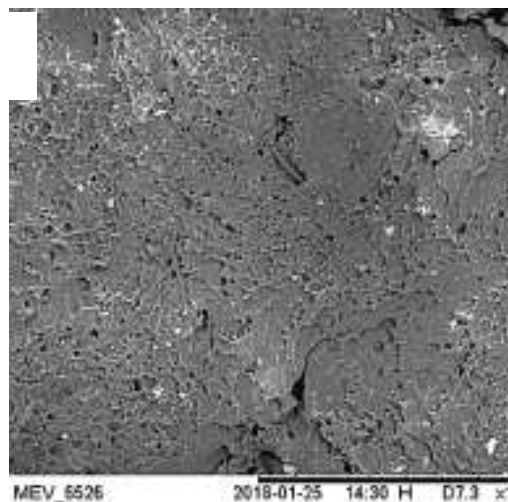

GM 1

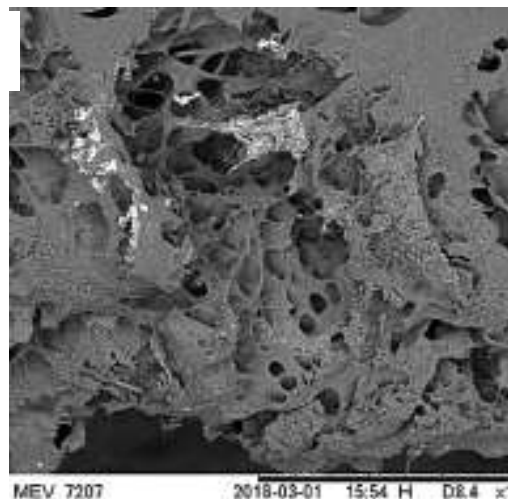

GM 2

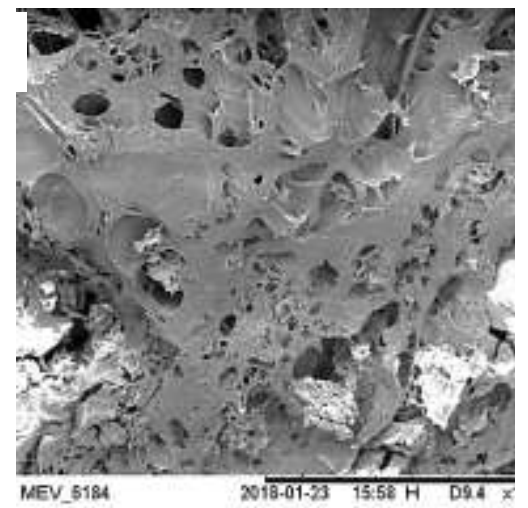

GM 3

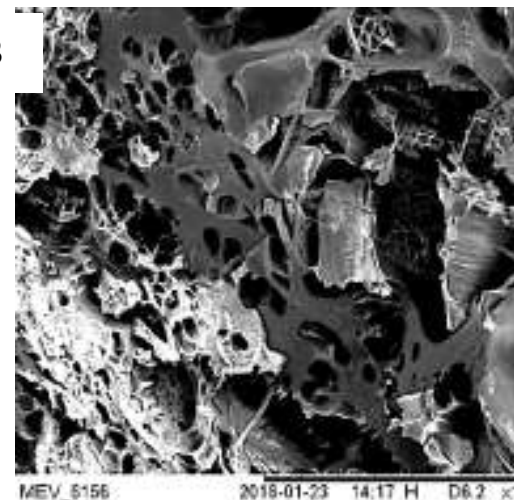

Fonte: Autores. 
Research, Society and Development, v. 10, n. 8, e12710817034, 2021

(CC BY 4.0) | ISSN 2525-3409 | DOI: http://dx.doi.org/10.33448/rsd-v10i8.17034

Figura 5. Micrografias das farinhas de trigo e glútens controle (FC e GC) e tratados por micro-ondas com potências de 100 W (FM 1 e GM 1), 450 W (FM 2 e GM 2) e 750 W (FM 3 e GM 3) glútens observadas por microscópio óptico: a) sem luz polarizada com aumento de 1000x; b) com luz polarizada e aumento de 1000x; c) fonte de luz padrão externa com aumento de 40x.

(a)

FC

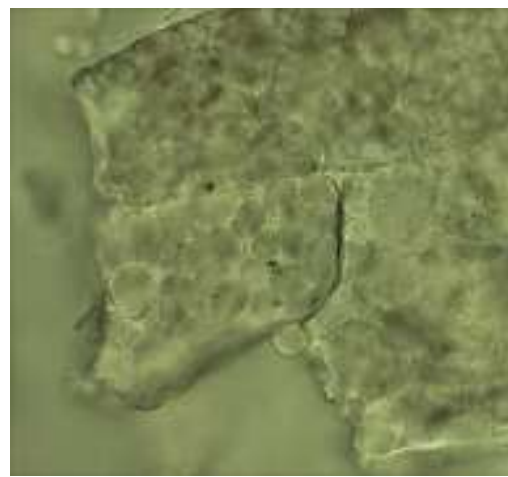

FM 1

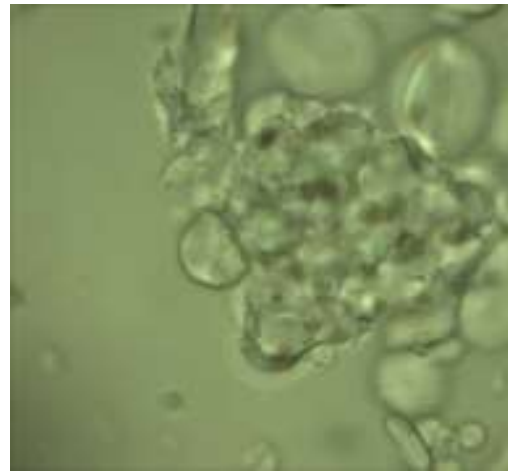

FM 2

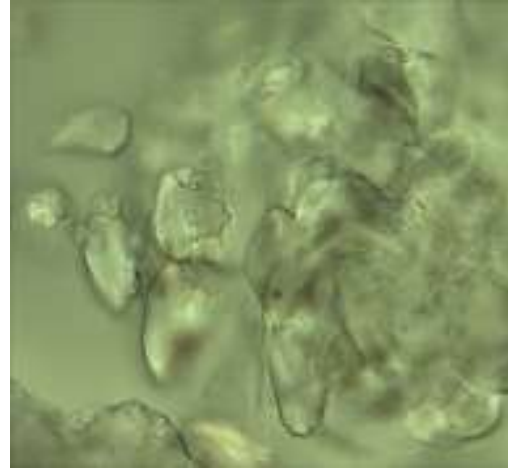

FM 3

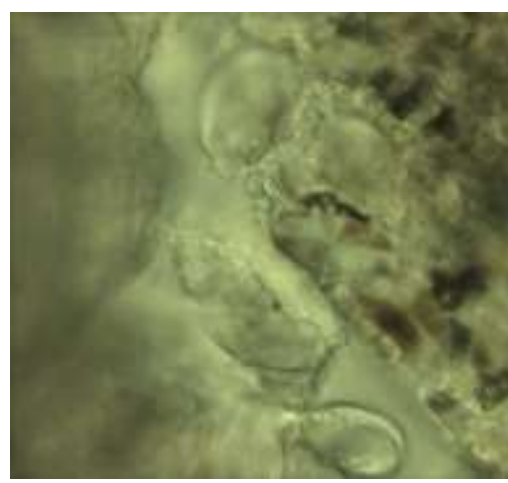

(b)
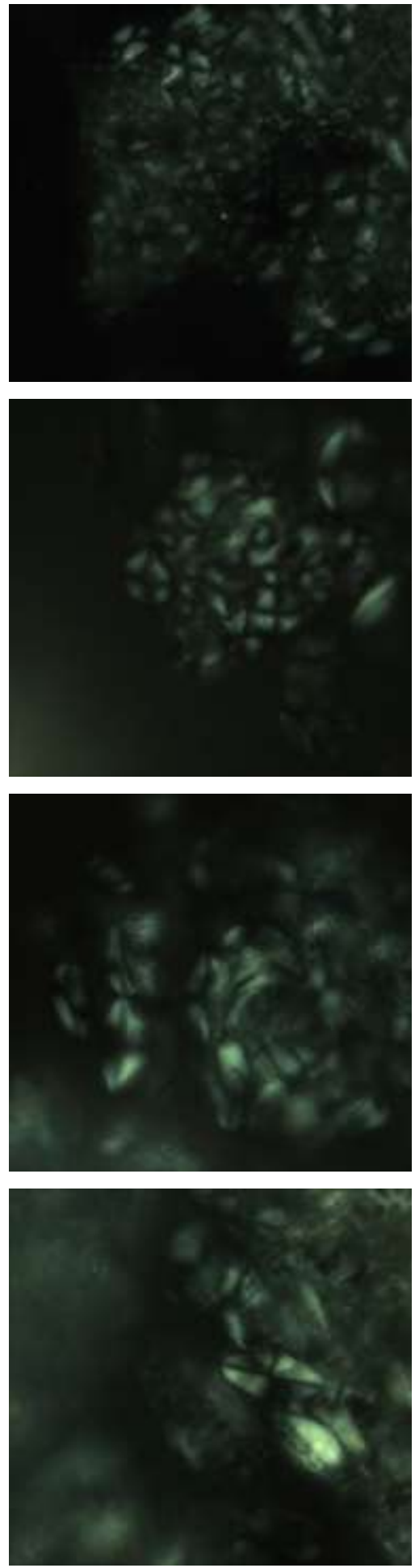

(c)
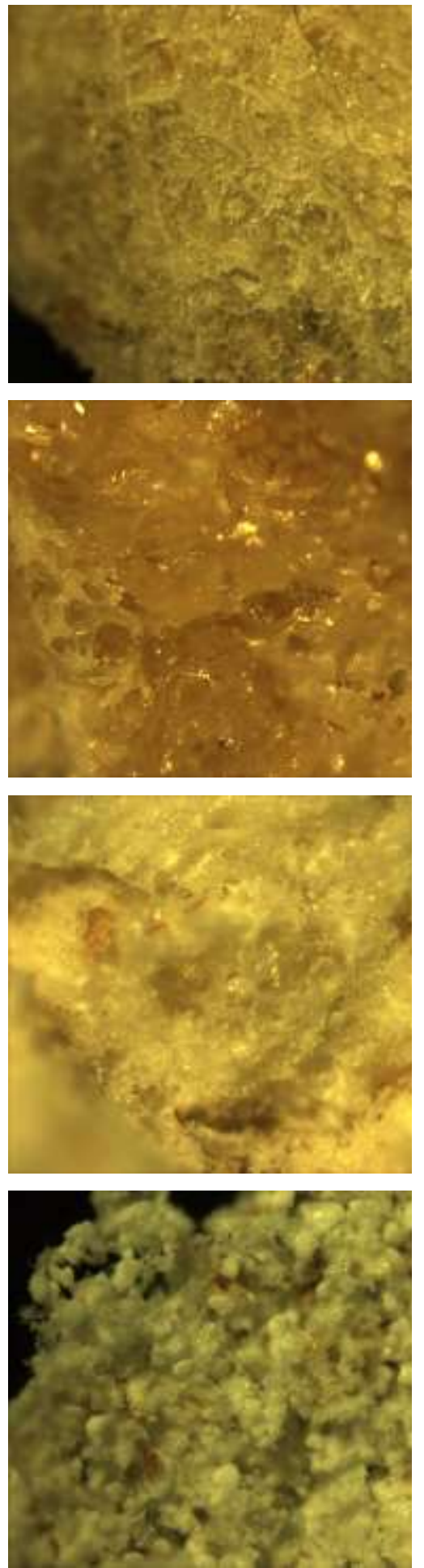

Fonte: Autores. 


\section{Conclusão}

As morfologias do grão do trigo, farinha e glútens avaliados sofreram alterações significativas e o presente estudo permite uma melhor avaliação e compreensão sobre o nível de alteração que o processamento por micro-ondas aplicados aos grãos de trigo, podem ocasionar às suas farinhas e respectivos glútens. As análises de cor e imagem podem complementar os resultados obtidos através das determinações físicas, físico-químicas e reológicas, normalmente utilizadas para qualificação de matérias primas e produtos finais.

\section{Agradecimentos}

Dedicamos este trabalho ao Prof. Dr. Antonio Marsaioli Junior (In memorian) pelo seu incansável trabalho para o desenvolvimento da tecnologia de alimentos no Brasil. Os autores são gratos à CAPES, Coordenação de Aperfeiçoamento de Pessoal de Nível Superior - Brasil (CAPES) - código de financiamento 001, ao Instituto de Tecnologia de Alimentos, ao Moinho Arapongas S.A. pela doação do trigo utilizado no presente trabalho, a Fundação de Amparo à Pesquisa do Estado de São Paulo (FAPESP) pelo apoio financeiro (Processo 2014/14435-1) e ao Conselho Nacional de Desenvolvimento Científico e Tecnol[ogico (CNPq) pela bolsa concedidas (150826/2019-2, 150826/2019-2 e 312786/2020-4).

\section{Referências}

AACCI. (2010). Approved Methods of Analysis. AACC International.

Adekunte, A. O., Tiwari, B. K., Cullen, P. J., Scannell, A. G. M., \& O’Donnell, C. P. (2010). Effect of sonication on colour, ascorbic acid and yeast inactivation in tomato juice. Food Chemistry, 122(3), 500-507. https://doi.org/10.1016/j.foodchem.2010.01.026

Aulakh, J., \& Regmi, A. (2013). Post-harvest food losses estimation-development of consistent methodology. First Meeting of the Scientific Review Committee of the Food and Agricultural Organization of the UN, 2050, 1-34.

Baloch, U. K. (2001). WHEAT: Post-harvest Operations (D. Mejia \& B. Lewis (eds.)). FOOD AND AGRICULTURE ORGANIZATION OF THE UNITED NATIONS.

Bansal, N., Dhaliwal, A. S., \& Mann, K. S. (2015). Dielectric properties of corn flour from 0.2 to 10 GHz. Journal of Food Engineering, 166, 255-262. https://doi.org/10.1016/j.jfoodeng.2015.06.019

Berteli, M. N. (2005). Estudo Comparativo de Processos de Secagem de Sólidos Granulados com e Sem Assistência de Microondas. Universidade Estadual de Campinas - UNICAMP.

Cauvain, S. (2015). Technology of breadmaking. In Technology of Breadmaking. Springer International Publishing. https://doi.org/10.1007/978-3-319-14687-4

Clerici, M. T. P. S., Flávio Ma, Marsaioli Junior, A., \& Bertelli, M. N. (2019). EQUIPAMENTO PROCESSADOR POR MICRO ONDAS PARA TRATAMENTO DE MATERIAIS ALIMENTÍCIOS (Patent No. BR 102019027383 6).

Ferreira, D. F. (2011). Sisvar: Um sistema computacional de análise estatística. In Ciencia e Agrotecnologia (Vol. 35, Issue 6, pp. 1039-1042). https://doi.org/10.1590/S1413-70542011000600001

Hagstrum, D. W., Phillips, T. W., \& Cuperus, G. (2012). Stored Product Protection (D. W. Hagstrum, T. W. Phillips, \& G. Cuperus (eds.)). Kansas State University Agricultural Experiment Station and Cooperative Extension Service.

Hemis, M., Singh, C. B., \& Jayas, D. S. (2011). Microwave-Assisted Thin Layer Drying of Wheat. Drying Technology, 29(10), 1240-1247. https://doi.org/10.1080/07373937.2011.584999

Hidalgo, A., Fongaro, L., \& Brandolini, A. (2017). Colour screening of whole meal flours and discrimination of seven Triticum subspecies. Journal of Cereal Science, 77, 9-16. https://doi.org/10.1016/j.jcs.2017.07.006

Kumar, D., \& Kalita, P. (2017). Reducing Postharvest Losses during Storage of Grain Crops to Strengthen Food Security in Developing Countries. Foods, 6(1), 8. https://doi.org/10.3390/foods6010008

Lamacchia, C., Landriscina, L., \& D’Agnello, P. (2016). Changes in wheat kernel proteins induced by microwave treatment. Food Chemistry, 197, 634-640. https://doi.org/10.1016/j.foodchem.2015.11.016

Landriscina, L., D’Agnello, P., Bevilacqua, A., Corbo, M. R., Sinigaglia, M., \& Lamacchia, C. (2017). Impact of gluten-friendly ${ }^{\mathrm{TM}}$ technology on wheat kernel endosperm and gluten protein structure in seeds by light and electron microscopy. Food Chemistry, 221, 1258-1268. https://doi.org/10.1016/j.foodchem.2016.11.031

Patil, S. S., Kar, A., \& Mohapatra, D. (2016). Stabilization of rice bran using microwave: Process optimization and storage studies. Food and Bioproducts 
Research, Society and Development, v. 10, n. 8, e12710817034, 2021

(CC BY 4.0) | ISSN 2525-3409 | DOI: http://dx.doi.org/10.33448/rsd-v10i8.17034

Processing, 99, 204-211. https://doi.org/10.1016/j.fbp.2016.05.002

Vadivambal, R., Jayas, D. S., \& White, N. D. G. (2007). Wheat disinfestation using microwave energy. Journal of Stored Products Research, 43(4), 508-514. https://doi.org/10.1016/j.jspr.2007.01.007 